\title{
«LAS CLASES SIEMPRE VAN DE AQUÍ PARA ALLÁ»: PERMANENCIAS Y CAMBIOS EN LOS ESPACIOS PARA LA ESCUELA PRIMARIA EN RIO GRANDE DO SUL, BRASIL (1883-1928)
}

\author{
"Classes always go from here to there": continuities and \\ transformations in the spaces designed for primary schools \\ in Rio Grande do Sul/Brazil (1883-1928)
}

\section{Tatiane de Freitas Ermel ${ }^{\alpha}$}

Fecha de recepción: 16/04/2020 • Fecha de aceptación: 06/10/2020

Resumen. El Estado de Rio Grande do Sul/Brasil, siguiendo las pautas y modelos internacionales relacionados con la implementación, difusión y mejoras de la educación primaria, emprendió, entre fines del siglo XIX y las primeras décadas del XX, algunas iniciativas en torno a la necesidad de construcción de edificios escolares. El objectivo de este artículo es analizar las ideas y acciones que marcaron las permanencias y transformaciones emprendidas por la autoridad pública estatal en el espacio escolar, utilizando un modelo de construcción escolar, de 1883; un diseño estándar de 1899; un modelo de escuela rural de 1919 y un concurso de construcción de escuelas, de 1928. La investigación documental, de carácter historiográfico, toma como fuentes primarias los informes de las Direcciones de Educación Pública y Obras Públicas del Gobierno del Estado de Rio Grande do Sul. Constatamos la permanencia durante estas décadas de algunos aspectos, especialmente, relacionados con la necesidad de impuestos destinados a la construcción de escuelas, su ubicación en ciudades y pueblos, cuestiones de higiene, el lugar de residencia del profesor y la enseñanza graduada. Entre las principales transformaciones, destacamos la proyección de salas adaptables y esquinas redondeadas, educación mixta, salas para diferentes actividades, como un museo, laboratorios, administración, biblioteca y espacio para cine y radio escolar. Estas nuevas perspectivas del Estado estaban parcialmente alineadas con los principios de la Escuela Nueva, que se introdujeron en

\footnotetext{
${ }^{\alpha}$ Dpto. de Estudios Educativos. Facultad de Educación. Universidad Complutense de Madrid. Rector Royo Villanova, sn. 28040 Madrid. España. tdefreit@ucm.es (D) http://orcid.org/0000-0003-2002-5101.

Cómo citar este artículo: Ermel, Tatiane de Freitas. "Las clases siempre van de aquí para allá": permanencias y cambios en los espacios para la escuela primaria en Rio Grande do Sul, Brasil (1883-1928)». Historia y Memoria de la Educación 13 (2021): 149-178
} 
Brasil, especialmente durante la década de 1920, y el espacio escolar debería adaptarse al lugar central que ocuparía el niño en el proceso educativo, alineado con sus necesidades y curiosidades.

Palabras clave: espacio escolar; escuela primaria; Brasil; Escuela Nueva; edificios escolares.

Abstract. The state of Rio Grande do Sul/Brazil, following the international guidelines and models related to implementation, dissemination and improvements of primary education, undertook, between the end of the 19th century and the first decades of the 20th century, initiatives that responded to the need for the construction of school facilities. The objective of this paper is to analyze the ideas and actions that marked the continuities and the transformations related to government intervention in school spaces. For this purpose we will use a model of a school building, from 1883; a standard design project, from 1899; a model of an agricultural school, from 1919; and a competition for school constructions, from 1928. The documentary research, of a historiographical character, uses as primary sources the reports by the Direction of Public Instruction and Public Works of the State of Rio Grande do Sul. The paper shows the permanence throughout these decades of certain aspects, especially those concerning the need for tax revenue to pay for the building of schools, their location in the cities and villages, hygienist issues, the teachers' places of residence, and the graded school system. Among the main transformations, we highlight the design of cornerless, adaptable rooms; mixed classes; rooms for different activities, such as museums, laboratories, administrative rooms, libraries, and spaces for school movie theaters and radio stations. These new state prescriptions were partially aligned with the New School principles, which were introduced in Brazil especially throughout the decade of 1920; in keeping with this movement, it was thought that the design of school spaces should consider the central role that children would play in the educational process, taking into account their needs and curiosities.

Keywords: school space; primary school; Brazil; New School; building school.

\section{INTRODUCCIÓN}

La escuela moderna ha estado marcada, entre otras cosas, por la constitución difusa y permeable de sus espacios. Progresivamente, desde la configuración de los sistemas educativos nacionales occidentales, fue incorporando construcciones teóricas y estructurales que, en algunos casos, suponían continuidades o actualizaciones de lo existente, y en otros, rupturas y transformaciones. Unas y otras son las que se 
analizarán y discutirán en este artículo, desde las perspectivas de la forma escolar ${ }^{1}$ y de los elementos constituyentes de la cultura escolar ${ }^{2}$ problematizando, especialmente, los asuntos relativos a los espacios escolares, tales como su funcionalidad y las representaciones simbólicas de la educación que proyectaban. ${ }^{3}$

En Estados Unidos y en gran parte de los países de Europa y America Latina, entre la segunda mitad del siglo XIX y las primeras décadas del siglo $\mathrm{XX}$, las demandas pedagógicas, la higiene y la estética se alinearon con el ideal de escuela primaria, que además del objetivo de alfabetización, incorporaba una concepción de formación integral, con fines intelectuales, morales y fisicos. Separada de las demás instituciones a las que, hasta entonces, estaba ligada, como las iglesias y administraciones públicas locales, la construcción de un edificio propio dotó a la escuela de una identidad particular, y la monumentalidad de algunos de estos, en diferentes ciudades, representó un significativo emprendimiento de propaganda visual, como sucedió, por ejemplo, con la instauración del régimen republicano, que impulsó la creación de los primeros grupos escolares en Brasil, en 1889 y 1893 , respectivamente. ${ }^{4}$

\footnotetext{
${ }^{1}$ Guy Vincent (ed.), L'éducation prisonnière de la forme scolaire? Scolarisation et socialisation dans les sociétés industrielles (Lyon: Presses Universitaires de Lyon, 1994).

2 Dominique Julia, «La culture scolaire comme objet historique», Paedagogica Historica, 31 (1995): 353-382. https://doi.org/10.1080/00309230.1995.11434853 (consultado el 10-03-2017). André Chervel, La culture scolaire: une approche historique (Paris: Belin, 1998). Antonio Viñao Frago, Sistemas Educativos, culturas escolares y reformas. Razones y propuestas educativas (Madrid: Ediciones Morata, 2002). En Brasil, investigadores traen reflexiones importantes sobre la cultura escolar: Luciano Mendes Faria Filho et al. "A Cultura Escolar como categoria de análise e como campo de investigação na história da educação brasileira» Revista Educação e Pesquisa, 30, n. ${ }^{\circ} 1$ (2004): 139-159. Rosa Fátima Souza y Vera Teresa Valdemarin (Ed.), A Cultura Escolar em Debate: questões conceituais, metodológicas e desafios para a pesquisa (Campinas: Autores Associados, 2005). Diana Vidal, «Sobre cultura escolar e História da Educação: questões para debate», en História das culturas escolares no Brasil, eds. Diana Vidal y Cleonara Maria Schwatz (Vitória: EDUFES, 2010), 13-35.

${ }^{3}$ Antonio Viñao Frago, «Do espaço escolar e da escola como lugar: propostas e questões» en Currículo, espaço subjetividade: a arquitetura como programa, eds. Antonio Viñao Frago y Agustín Escolano Benito (Rio de Janeiro: DP\&A, 1998), 59-140.

${ }^{4}$ Entre finales del siglo XIX y las primeras décadas del siglo XX, los grupos escolares se convirtieron en el modelo de escuela primaria en la mayoría de los estados de Brasil. Consistieron en la reunión o agrupación de escuelas existentes en un área determinada de la ciudad (clases aisladas, que se regían solo por un maestro). El término «escuela» deja de usarse solo para designar a un grupo de estudiantes y ahora se refiere a un espacio especializado, con características apropiadas para su función de enseñanza-aprendizaje. La denominación de Grupo Escolar cambió con la Ley n. ${ }^{\circ} 5$ 692, de diciembre de 1971, que transformó la educación primaria en primer grado, de 8 años. Rosa Fátima Souza, Tempos de Civilização: a implantação da Escola Primaria Graduada no Estado de São Paulo (1890-1910), (São Paulo: UNESP, 1998).
} 
La escuela primaria estuvo vinculada a tramas discursivas amplias, que facilitó que fuera incorporando parte de los cambios producidos en diferentes ámbitos de la sociedad, entre los que se pueden destacar dos: por un lado, los procesos de reconfiguración de las ciudades y los discursos higienistas de finales del siglo XIX e inicios del XX, y, por otro lado, la utilización de aquella como soporte de signos y ornamentos con una fuerte carga simbólica -banderas, escudos patrios, relojes, cuadros murales, símbolos religiosos, figuras políticas, etc.-, visibles tanto en las fachadas como en los interiores de los edificios. ${ }^{5}$

Algunas recomendaciones y prescripciones sobre espacios escolares fueron compartidas por diferentes países y regiones a través de las Exposiciones Universales y Conferencias Pedagógicas, así como de los contactos de políticos y educadores con experiencias de diferentes países.

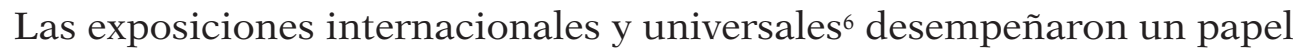
importante en la difusión y circulación de conocimientos y novedades relacionadas con la educación y, más específicamente, la enseñanza. Aunque comenzaron en Londres, en 1851, no fue hasta la Exposición de París de 1867 cuando se presentaron cuestiones relacionadas con los planos de clase y las reconstrucciones. Fue durante esos años, en un contexto de progresiva superación del sistema de enseñanza mútua, cuando se publicaron en Europa (Francia, 1858; Suiza, 1861; Portugal, 1866; Bélgica 1874) las primeras normas para edificios escolares, centrándose en «uma nova ordem pedagógica, isto é, na 'classe' como agrupamento de alunos (e como sala)». ${ }^{7}$

Tales normas, desde el principio, conllevaron la discusión de asuntos vinculados a la organización de los espacios escolares que trascedían las cuestiones estrictamente arquitectónicas. Los más comunes y persistentes fueron, principalmente, los siguientes: la separación o no de la casa del profesor del espacio escolar, que se resolvió de distinta manera, dependiendo de si se trataba de entornos urbanos o rurales; la enseñanza

\footnotetext{
${ }_{5}^{5}$ Agustín Escolano Benito, Tiempos y espacios para la escuela. Ensayos Históricos (Madrid: Biblioteca Nueva, 2000).

6 Sobre la Primera Exposición de Rio de Janeiro, véase: Maria Helena Camara Bastos «A educação como espetáculo», en Histórias e Memórias da Educação no Brasil, eds. Maria Stephanou y Maria Helena Camara Bastos (Petrópolis: Vozes, 2005), 116-131.

${ }^{7}$ Carlos Manique da Silva, Escolas belas ou espaços sãos? Uma análise histórica sobre arquitetura escolar portuguesa (1860-1920) (Lisboa: Instituto de Inovação Educacional, 2002$), 15$.
} 
graduada, que preveía aulas para diferentes niveles de educación, en función de la edad de los estudiantes; las agrupaciones en función del sexo, que exigía, entre otras cosas, escuelas independientes y/o salones separados para niños y niñas; la preocupación higienista, que hacía hincapié en la necesidad de estructuras que permitieran la circulación del aire y la entrada de luz en las aulas para evitar el surgimiento y la propagación de enfermedades; los aspectos económicos, dado que los edificios, ya fueran alquilados, adaptados o de nueva construcción, requirieron de considerables inversiones por parte de las autoridades públicas; los espacios para el desarrollo de actividades físicas y/o al aire libre; la relación con el entorno escolar y la representación política, social y cultural de la escuela, que se sometió a un proceso de valorización de las instituciones públicas e incorporó, en muchos casos, más allá de la monumentalidad/suntuosidad, un conjunto de símbolos y signos que la han demarcado como un lugar específico y reconocido en la trama de ciudades y pueblos. ${ }^{8}$

La generalizada precariedad de los espacios escolares existentes o la falta de estos hicieron que algunos países los regularan, establecieran modelos y abrieran concursos de edificios escolares, ajustados a lo que consideraban entornos y edificios ideales para los fines y estilos de enseñanza y aprendizaje por los que apostaban. En el entorno europeo, destacan los casos de España, Portugal y Francia. En este último país, en 1890, el arquitecto francés Georges Tubeuf publicó un tratado que examinó las nuevas cuestiones educativas. Su trabajo consistió, por un lado, en la compilación de leyes, decretos y circulares promulgados en Francia entre 1850 a 1887, con el fin de anunciar un nuevo tiempo para la discusión de la educación pública, entendida como un derecho, y para incentivar al Estado para que estableciera normas para los edificios escolares; 9 por otro lado, también analizó la arquitectura escolar que se estaba desarrollando en otros países, entre los que se contaron Bélgica, Alemania, Suiza, Suecia, Noruega, Dinamarca, Grecia, Rusia, Italia,

\footnotetext{
${ }_{8}^{8}$ Tatiane de Freitas Ermel, «Arquitetura escolar e patrimônio histórico-educativo: os edifícios para a escola primária no Rio Grande do Sul» (Tesis doctoral, Pontifícia Universidade Católica do Rio Grande do Sul, 2017).

9 Ângelo Marques Vieira de Arruda, «Arquitetura dos edifícios da escola pública no Brasil (18701930): construindo os espaços para a educação» (Tesis doctoral, Universidade Federal do Mato Grosso do Sul, 2010), 48. La obra de referencia de Georges Tubeuf es: Traité d'architecture théorique et pratique. Tome IV, Tipes de constructions diverses: edifices publics et divers (Paris: Georges Fancho, Editeur, 1890).
} 
Austria, Inglaterra, Portugal y España. A pesar de la variedad de opciones que presentó, la idea del arquitecto francés era lograr una escuela tipo, es decir, una construcción escolar que pudiera eregirse en distintos lugares, pero siguiendo un único proyecto arquitectónico.

En Portugal hubo dos momentos clave para el desarrollo de la arquitectura escolar. El primero fue el Congreso Pedagógico de Lisboa, celebrado en 1897, que dedicó especial atención a los edificios que albergaban las escuelas. El segundo fue el plan de construcción de las escuelas primarias Adães Bermudes, puesto en marcha en 1898, bajo la responsabilidad de la Dirección Técnica de Edificios Escolares; en esta ocasión, se abrió un concurso público, a cargo de la Asociación de Ingenieros Civiles Portugueses, que concedió un peso específico a los intentos de adaptar al contexto portugués ideas y modelos de arquitectura escolar desarrollados en otros países. ${ }^{10}$

En España, tras la creación del Ministerio de Instrucción Pública (1900) y la Oficina de Arquitectura (1904), tales asuntos cobraron cierto protragonismo, plasmándose, entre otros modos, en proyectos de ampliación de edificios escolares por todo el territorio y concursos de edificios que se ajustaran a las nuevas exigencias de la reforma pedagógica de las escuelas primarias, progresivamente transformadas en escuelas graduadas, en las que los espacios debían estar preparados para la separación de los alumnos por edades y grados de cultura. ${ }^{11}$

En Estados Unidos, durante la segunda mitad del siglo XIX, se pasó, progresivamente, de pequeños edificios escolares, con áreas restringidas y carentes de jardines y espacios abiertos, a construcciones que contemplaban espacios amplios y abiertos, edificios arquitectónicamente modernos y exuberantes, así como la incorporación de otros elementos novedosos, como auditorios y gimnasios. Como señala Upitis al proponer un análisis de la arquitectura de la escuela y su complejidad en la sociedad contemporánea, ${ }^{12}$ las cuestiones como la importancia de la luz y la

\footnotetext{
10 Manique, Escolas belas ou espaços sãos?

11 José Miguel Godínez Visedo, «Legislación comentada sobre espacios y construcciones escolares de los centros públicos primarios en España. De 1825 a 1991» Anales de Pedagogía 11 (1993): 229-243.

12 Rena Upitis, "School Architecture and Complexity», Complicity: An International Journal of Complexity and Education, 1 (2004):19-38. https://doi.org/10.29173/cmplct8713 (consultado el 10-062020).
} 
ventilación, el mobiliario adecuado y la necesidad de espacios verdes ya fueron reconocidos como esenciales por el primer Comisionado de Educación de los Estados Unidos, Henry Barnard (1811-1900), en su obra clásica sobre los espacios escolares, en la segunda mitad del siglo XIX.13

A inicios del siglo XX, el estadounidense Frank Lloyd Wright, precursor de la arquitectura orgnánica, incorporó elementos importantes de la arquitectura civil a la escolar, entre los que destacaron los relacionados con la planificación de espacios, los detalles de calidad, la estética y el bienestar de los usuarios, utilizando los presupuestos funcionalistas y racionalistas. Así, apostó por edificios predominantemente horizontales, anchos y planos, como si la estructura creciera naturalmente desde el interior de un espacio. ${ }^{14}$

En América Latina, las discusiones sobre las construcciones escolares también abordaron asuntos relativos a técnicas, materiales y estructruras, pero añadieron otros, como el higienismo, que fue una de las cuestiones que mayor atención captó. Así, por ejemplo, el Congreso Pedagógico de América del Sur, celebrado en Argentina en 1882, se centró en la dotación de utillaje escolar y los problemas sanitarios -especialmente, el acceso al agua- vinculados a los espacios escolares, ${ }^{15}$ y el gobierno de México, en 1905, creó una Junta de Directiva de Obras de Edificios Primarios, con el propósito de satisfacer las demandas sanitarias y pedagógicas, que prestó especial atención a los lugares y terrenos donde se instalarían las escuelas: se estableció una distancia mínima de cien metros desde lugares insalubres, peligrosos o ruidosos, y que la zona urbana tuviera acceso a servicios de saneamiento y, a ser posible, al sistema de drenaje publico. ${ }^{16}$

\footnotetext{
${ }_{13}$ Henry Bernard, School Architecture: contributions to the improvement of school-houses (Cincinati: Published by H.W. Derby and Co., 1855).

${ }^{14}$ Arruda, Arquitetura dos edifícios da escola pública no Brasil, 74-75. Para un estudio de la historia de los edificios de escuelas públicas en los Estados Unidos, véase: Amy S. Weisser, «Little Red School House, What Now? Two Centuries of American Public School Architecture», Journal of Planning History, 3 (2006): 196-217. https://doi.org/10.1177/1538513206289223 (consultado el 10-06-2020)

${ }_{15}$ Héctor Recalde, El Primer Congreso Pedagógico/1 (1882), (Biblioteca Política Argentina: Centro Editor de América Latina, 1987), 112.

16 Carlos Ortega Ibarra, "Ciencia y revolución en la arquitectura escolar. Ciudad de México (19101920)» Revista Ciencia y Desarrollo, (2012), 39-42.
} 
En Brasil, tras la proclamación de la República (1889) y la subsiguiente promulgación de la Constitución (1891), se consolidó lo que prevéia el Acto Adicional de 1834, que estableció que cada Estado tendría la responsabilidad de crear y supervisar su red de educación primaria (República Federativa). ${ }^{17}$ De este modo, los diferentes Estados del país tuvieron que desarrollar sus propias regulaciones y crear modelos para la construcción de edificios escolares; en este proceso destacó el espíritu pionero de São Paulo, que, desde 1893, puso en marcha los primeros grupos escolares y albergó las novedosas experiencias de escuela graduada.

Sin embargo, vale la pena mencionar algunas experiencias aisladas, pero de gran importancia simbólica, de la construcción de edificios escolares que se emprendieron en el período del Segundo Imperio Brasileño (1840-1889). En la capital, la ciudad de Río de Janeiro, entre 1870 y 1877 , se construyeron ocho escuelas públicas, financiadas por el gobierno y/o por iniciativa privada, pero entregadas a la administración pública después de la finalización de las obras. ${ }^{18}$

En São Paulo, la Ley de Educación Obligatoria n. ${ }^{\circ}$ 9, de 22 de marzo de 1874, fue desarrollada por la Ley n. ${ }^{\circ} 55$, del 30 de marzo de 1876, que determinó los criterios y estableció las normas para la construcción de edificios escolares. En la práctica, las instituciones públicas de educación primaria continuaron funcionando, principalmente, en espacios ya existentes, pero adaptados por el Estado. Un ejemplo significativo de esto es la construcción de la Escuela Pública Arouche, realizada como un proyecto para el Departamento de Obras Públicas de São Paulo, concluido en 1877. Su inauguración, recogida en la prensa diaria de junio

\footnotetext{
17 Carlos Roberto Jamil Cury, «A educação e a primeira constituinte republicana», en Educação nas constituições brasileiras 1823-1988, ed. Osmar Fávero (Campinas: Autores Associados, 1996), 69-80.

18 Silvia Ferreira Santos Wollf, Escolas para a República: os primeiros passos da Arquitetura das escolas públicas paulistas, 82. Para un estudio sobre el proceso de expansión de las escuelas primarias en Río de Janeiro entre los años 1870 y 1906, con énfasis en regiones consideradas rurales o periféricas, véase: Alessandra Frota Schueler y Irma Rizzini, «Entre becos, morros e trilhos: expansão da escola primária na cidade do Rio de Janeiro (1870-1906)» Cadernos de História da Educação 18, n. ${ }^{\circ} 1$ (2019): 160-175. https://doi.org/10.14393/che-v18n1-2019-9 (consultado 14-07-2020). Sobre la influencia extranjera en los programas arquitectónicos brasileños en el período imperial, véase: Francisca Maria Teresa dos Reis Baltar, «Arquitetura de escolas no século 19: primeiras escolas construídas no Brasil», Revista História da Educação, n. 10 (2001): 53-84. https://seer.ufrgs.br/asphe/article/view/30525/pdf (consultado el 03-07-2020).
} 
de ese mismo año, presentó el edificio de la escuela como «(...) modesto pero elegante». ${ }^{19}$

Las construcciones de edificios de escuelas primarias públicas del Estado de São Paulo, realizadas durante los inicios de la Primera República Brasileña, tuvieron la intención de difundir una imagen de estabilidad de las administraciones, que se materializó, en muchos casos, en la búsqueda de monumentalidad y en una preocupación excesiva por la visbilidad de las escuelas públicas, de modo que fueran «muito evidentes, facilmente percebidas e identificadas como espaços da ação governamental». ${ }^{20}$ Gran parte de los edificios fueron construídos siguiendo un mismo patrón, dejando en manos del arquitecto sólo el ornamento de las fachadas. El proyecto del Grupo Escolar de Campinas/São Paulo, diseñado por Ramos de Azevedo, funcionó como una especie de modelo para casi todos los demás construidos en el interior del Estado, entre finales del siglo XIX y principios del XX.21

En el Estado de Minas Gerais, el «Regimento interno de grupos escolares e escolas isoladas»-Decreto 1.969, del 3 de enero de 1907-puso las primeras reglas para el uso de espacios escolares. En 1910, el Gobierno publicó el compendio "Typos para a construção de prédios escolares», dirigido por José Dantas, donde los modelos adoptados fueron tres: escuela rural, escuela aislada y grupo escolar. El primero, con un solo salón de clases; el segundo, con dos aulas, y el tercero, con cuatro, seis u ocho aulas, además de otros compartimentos. ${ }^{22}$

Seguiendo las orientaciones internacionales y las regulaciones nacionales de elaboración de concursos, modelos y proyectos tipo de edificaciones escolares, el Estado de Rio Grande do Sul,23 de forma bastante más tímida que otros Estados pioneros, como el caso de São Paulo, emprendió, desde finales del siglo XIX, algunas iniciativas que marcaron su

\footnotetext{
19 Souza, Tempos de Civilização, 112-114.

20 Wollf, Escolas para a República, 59.

${ }^{21}$ Wollf, Escolas para a República, 35-162.

22 Marcus Levy Bencostta y Marina Fernandes Braga, «História e Arquitetura escolar: a experiência dos regulamentos franceses e brasileiros para os edifícios escolares (1890-1910)», Revista Linhas 12, n. ${ }^{\circ} 1$ (2011): 51-72.

${ }^{23}$ El Estado de Rio Grande do Sul integra la región Sur de Brasil, junto con los Estados de Santa Catarina y Paraná. Sus fronteras internacionales son Uruguay y Argentina.
} 
trayectoria en materia de construcciones escolares de nivel primario durante la Primera República Brasileña. El objetivo de este artículo es analizar las ideas y acciones que marcaron las permanencias y transformaciones emprendidas por la autoridad pública estatal para los espacios escolares, concretadas en el modelo de construcción escolar de 1883, en el diseño estándar de 1899, en el modelo de escuela rural de 1919 y en el concurso de construcción de escuelas de 1928. La investigación documental, de carácter historiográfico, toma como fuentes primarias los Informes anuales de las Direcciones de Educación Pública y Obras Públicas del Gobierno del Estado de Rio Grande do Sul. ${ }^{24}$

\section{MODELOS PARA LA ESCUELA PRIMARIA EN RIO GRANDE DO SUL}

En el Estado de Rio Grande do Sul, la escasez de edificios para instalar escuelas primarias fue un problema recurrente durante todo el período analizado. En 1929, Getúlio Vargas, siendo presidente del Estado de Rio Grande do Sul, reprodujo una intervención del político João Maia en Asemblea Legislativa, en la que rememoraba el año 1899 y señalaba que la instalación de casas devidamente construídas para las actividades de enzeñanza era un elemento indispensable para la estabilidad de las escuelas públicas en el Estado; según el propio Maia, «as aulas andam sempre daqui para acolá», lo que se traducía en una verdadeira contradança de los espacios escolares.

En este sentido, el entonces presidente recalcó que los viejos problemas persistían en el escenario educativo de Rio Grande do Sul, lo que significaba, entre otras cosas, que persistía la inestabilidad que representaban los espacios escolares adaptados, a pesar de haber pasado cuatro décadas desde la instauración del régimen republicano. Por lo tanto, la escuela primaria estaba lejos de cumplir con los requisitos que se habían ido presentando como programas arquitectónicos ideales, tanto en el extranjero como en otros Estados brasileños.

La primera propuesta conocida -hasta el momento- para la construcción de un edificio escolar específicamente pensado para educación

\footnotetext{
${ }^{24}$ Los Informes anuales de la Diretoria de Instrução Pública e Obras Públicas do Estado do Rio Grande do Sul, de 1889 hasta 1930, han sido consultados en el Arquivo Histórico do Rio Grande do Sul y en el Memorial da Assembleia Legislativa do Estado do Rio Grande do Sul, ambos ubicados en el centro histórico de Porto Alegre/Brasil.
} 
primaria en Rio Grande do Sul es anterior a la proclamación de la República Brasileña; data de 1883. Inspirado en el Reglamento de Instrucción Pública de Francia (1880), el edificio escolar debería albergar dos escuelas, una para niños y otra para niñas, en cuya planta superior se situaba la casa del maestro y de la maestra, que constaba de una cocina, un baño, dos dormitorios, una oficina, una sala de estar y dos balcones, uno a la izquierda, para el maestro, y otro a la derecha, para la maestra; los accesos a las aulas se hicieron por escaleras, para uso exclusivo de cada sexo. ${ }^{25}$

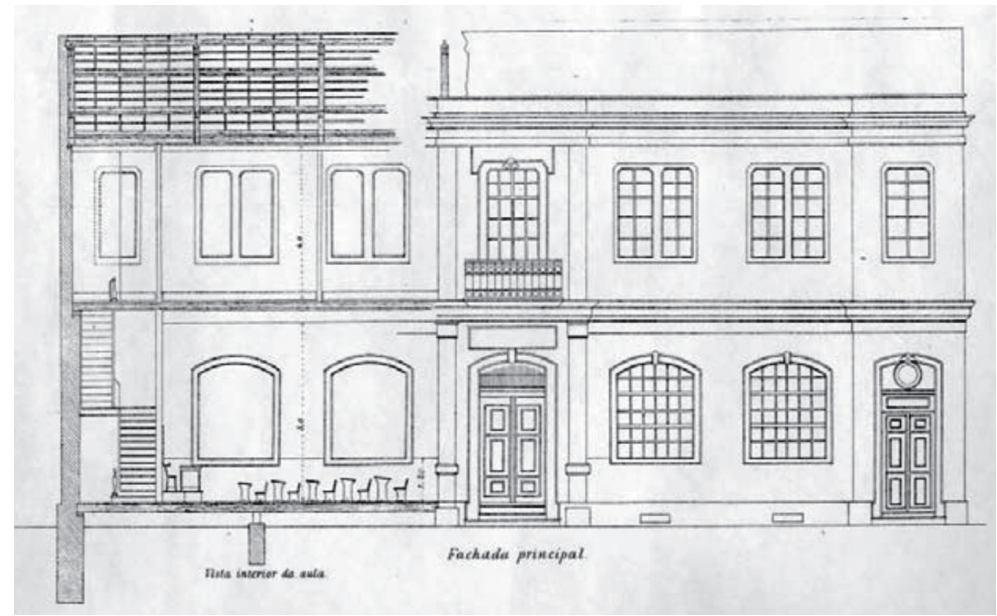

Imagen 1. Fachada del proyecto - tipo de escuela (1883). ${ }^{26}$

El proyecto contemplaba una fachada de veinticuatro metros de frente, con dos pisos, tres puertas y cuatro ventanas, siendo que «as salas de aula abriam-se para o exterior por quatro amplas janelas, duas voltadas para o pátio interno e duas na fachada exterior para a rua». ${ }^{27}$ Cada clase tenía espacio para recibir veinte estudiantes, que se acomodarían en filas, con pupitres individuales, frente la pizarra y la mesa del maestro. El patio tenía una pequeña área cubierta con equipos de gimnasia, y una pared dividía aquel en dos, separando, así, a los niños y las

\footnotetext{
25 Flávia Obino Corrêa Werle, «Instrução Pública e Configuração do Mundo Urbano», História da Educação, n. 18 (2005): 83-95. https://seer.ufrgs.br/asphe/article/view/29128 (consultado el 16-07-2020)

26 Fuente: Flávia Obino Corrêa Werle, «Instrução Pública e Configuração do Mundo Urbano», História da Educação 18 (2005), 89.
}

27 Werle, «Instrução Pública», 89. 
niñas; en ambos casos, las letrinas, distribuidas por sexos en igual número, se situaban al lado de la pared trasera del patio.

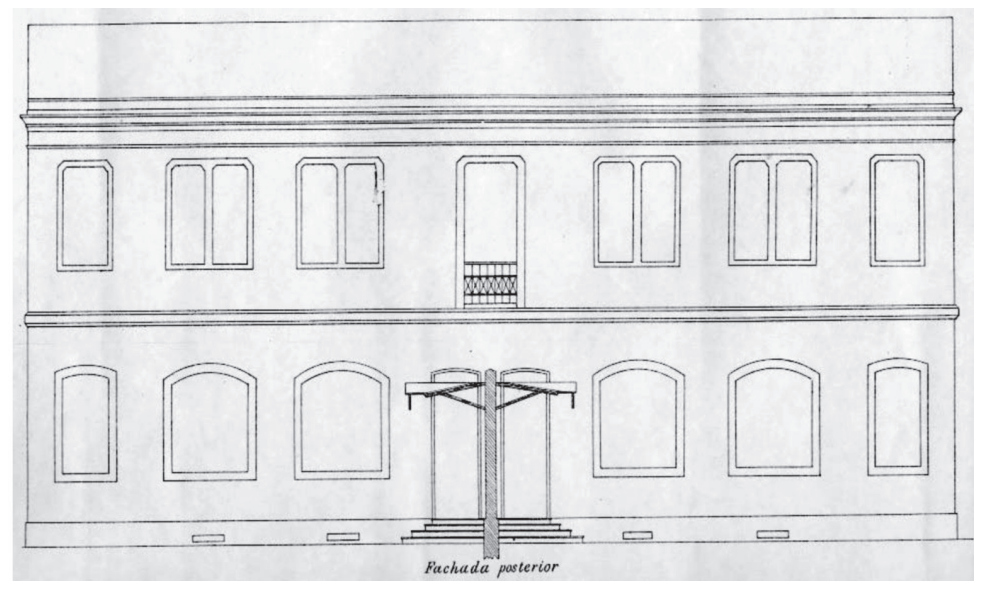

Imagen 2. Fachada interna de un edificio escolar de 1883.28

La última década del siglo XIX, ya en tiempos de la República, estuvo marcada por la necesidad de buscar fórmulas que permitieran al gobierno aumentar el número de escuelas primarias instaladas en edificios apropiados, algo que se consideraba un requisito indispensable para mejorar la educación allí ofrecida. Según la Dirección de Educación Pública, la progresiva sustitución de casas alquiladas por edificios diseñados y construidos específicamente para ser escuelas era necesaria por cuatro motivos, fundamentalmente: económico, pues el alquiler de espacios adaptados aumentaba significativamente el gasto; educativo, ya que en el diseño no incluyeron el número de estudiantes que habría y no pensaron en que habría que dividirlos en grados de enseñanza, como recomiendaba la pedagogía moderna; higiénico, dado que los espacios adaptados solían carecer de baños y las habitaciones eran pequeñas, tenían poca luz y mala circulación del aire; y material, puesto que el mobiliario destacaba por ser «inapropiado, antigo e sem elegância, parecendo atestar a nossa inacessibilidade aos progressos da Pedagogia». ${ }^{29}$

\footnotetext{
${ }^{28}$ Werle, «Instrução Pública e Configuração do Mundo Urbano», 91.

29 João Abbott «Relatório da Secretaria do Interior e do Exterior do Estado do Rio Grande do Sul, Diretoria de Instrução Pública», julio de 1896, p. 5. Memorial da Assembleia Legislativa do Estado do Rio Grande do Sul.
} 
En las discusiones previas sobre el Reglamento de Instrucción Pública, el director de Educación Pública del Estado de Rio Grande do Sul, Manoel Pacheco Prates, analizó, además de los problemas mencionados anteriormente sobre los edificios escolares, las ventajas de construir un edificio en centro de cada distrito, donde se reuniría a la población escolar:

espaçosos, onde deverão lecionar vários professores em graus diferentes de ensino, de modo a evitar-se a falta de método [...] Estas escolas terão, mais, a vantagem de servir de modelo para estabelecimentos congêneres, que pouca a pouco se irão fundando nos municípios. [...] Esta reforma terá geral aceitação e nós obteremos indubitavelmente a uniformização do ensino, partindo a medida do centro para a periferia.$^{30}$

Teniendo en cuenta estas consideraciones, el Reglamento de Educación Pública de 1897 estableció algunas prerrogativas para la organización de la red de escuelas primarias y, especialmente, para su división por zonas de inspección, pero careció de especificaciones sobre los espacios y los edificios destinados a la enseñanza. Dos años después, en 1899, se presentó el proyecto estándar (tipo) -Projeto-Typo para Escola Pública-, con un solo piso, con capacidad para sesenta estudiantes, sin sanitarios en el edificio y sin más prescripciones para los espacios escolares.

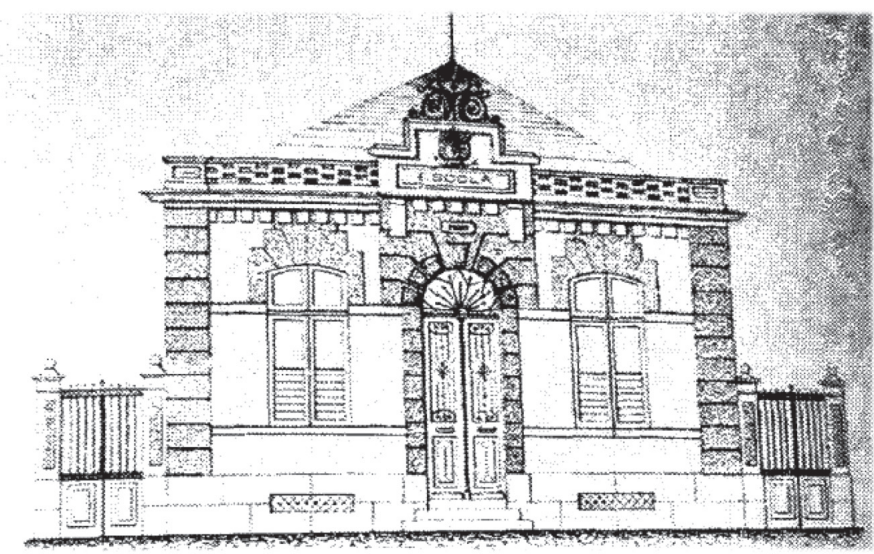

Imagen 3. Fachada del Projeto-Typo para Escola Pública (1899).31

\footnotetext{
30 João Abbott «Relatório da Secretaria do Interior e do Exterior», p. 6.

31 João Abbott «Relatório da Secretaria do Interior e do Exterior do Estado do Rio Grande do Sul, Diretoria de Instrução Pública», agosto de 1900, s/p. Memorial da Assembleia Legislativa do Estado do Rio Grande do Sul.
} 
Algunos años después, la Diretoria de Obras Públicas dio a conocer, a través de su Informe Anual de 1907, las necesidades de construcción de edificios públicos, entre ellos, de los específicos para instalar escuelas primarias en zonas urbanas y rurales, la escuela complementaria que serviría para la formacion de maestros-, las colonias para el tratamiento de los alienados y los centros penitenciarios, todos ellos adaptados a las necesidades y orientaciones de la ciencia moderna, uno de los pilares para el desarrollo material e intelectual. ${ }^{32}$

Ese mismo año, basánsode en el proyecto estandár de 1899, la Dirección de Obras Públicas comenzó la construcción de un edificio en la ciudad de Porto Alegre, la capital del Estado de Rio Grande do Sul. Como se puede ver en la figura siguiente (cuatro) y en las descripciones que se refieren a la organización interna y externa de sus espacios, la construcción, de ocho por doce metros y ochenta y cinco centímetros, tenía dos pisos, que no se corresponden exactamente con el diseño estándar, de 1899, de solo uno. El piso inferior podría usarse para actividades estudiantiles en días lluviosos o como casa del maestro. También se puede ver una escalera externa, que daba acceso al piso superior -a dos metros y veinte centimetros del suelo-, donde se desarrollarían las clases, con capacidad para aproximadamente sesenta estudiantes. Siguiendo la línea del proyecto que sirvió de inspiración, su fachada era simple, con solo la inscripción escuela, y una verja de hierro en el lateral del recinto donde se situaba el acceso principal una puerta de hierro-, que daba a la Avenida Redenção. Y, al igual que en la propuesta de 1899, no se mencionaron los sanitarios, que se encontraban, en forma de letrinas, en el exterior del edificio, al fondo del terreno. ${ }^{33}$

\footnotetext{
32 Protásio Antonio Alves «Relatório da Secretaria de Obras Públicas do Estado do Rio Grande do Sul», 1907, p. 2. Arquivo Histórico do Rio Grande do Sul (AHRGS).

33 Para un estudio sobre la trayectoria de este edificio, desde su construcción hasta 2016, véase: Tatiane de Freitas Ermel, «Transfigurações no tempo e no espaço: Aula Isolada Campo da Redenção em Porto Alegre/RS (1907-2016)», Revista Brasileira de História da Educação 18, n. ${ }^{\circ} 48$ (2018): 1-32, http://dx.doi.org/10.4025/rbhe.v18.2018.e006 (Consultado el 17-08-2020); Protásio Antonio Alves «Relatório da Secretaria de Obras Públicas do Estado do Rio Grande do Sul», 1907, p. 40.
} 


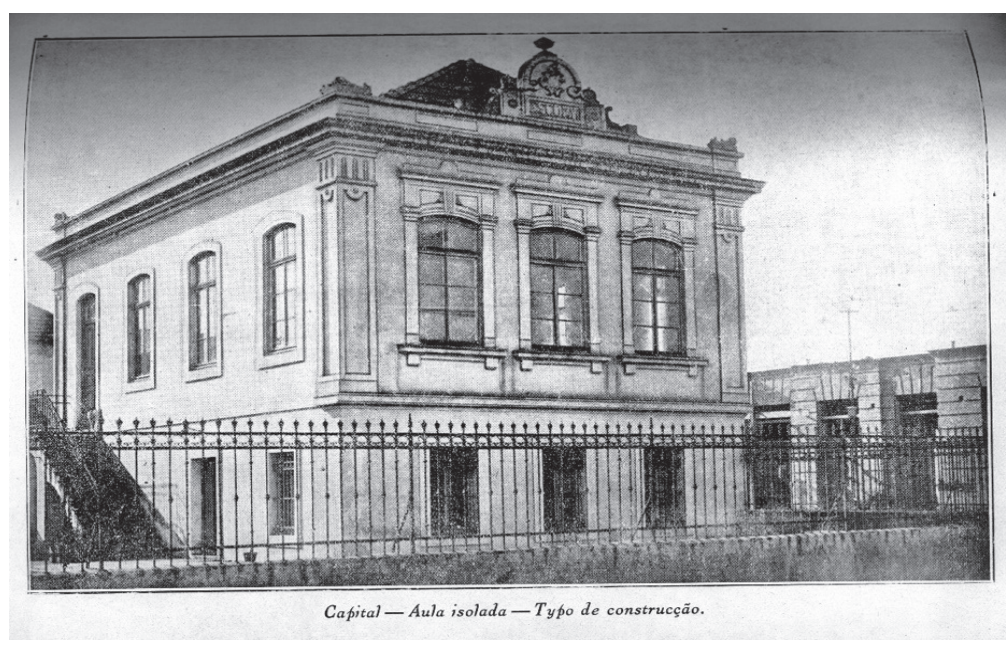

Imagen 4. Escuela Aislada Campos da Redenção (1924). ${ }^{34}$

Al igual que en la mayoría de las ciudades, las zonas rurales sufrían la carencia de construcciones escolares suficientes para atender a la población, pero esta realidad era agrabada por otras dificultades: materiales, carencia de medios de transporte y escaso número de estudiantes, muchas veces sin llegar al mínimo que justificara la inversión económica por parte del Gobierno. En el año 1919, la Dirección de Obras Públicas, en un intento de paliar tal situación, elaboró un plan estándar de escuela colonial, ${ }^{35}$ con algunos detalles y especificaciones sobre la organización del espacio escolar, que consitió en un modelo de madera, con planos, cortes longitudinales y transversales, fachada y diseño lateral.

\footnotetext{
${ }^{34}$ Protásio Alves, «Relatório da Secretaria do Interior e do Exterior do Estado do Rio Grande do Sul, Diretoria de Instrução Pública», setembro de 1924, s/p. Memorial da Assembleia Legislativa do Estado do Rio Grande do Sul.

35 Mantenemos la nomenclatura utilizada por el gobierno del Estado de «escuela colonial», es decir, un tipo de escuela primaria que fue construida por el Estado en zonas de inmigración de diferentes grupos étnicos. Para estudios específicos, véase: Lúcio Kreutz, «A representação de identidade nacional em escolas da imigração alemã no Rio Grande do Sul», Revista História da Educação 3, n. ${ }^{\circ}$ 5, (1999): 141-164. https://seer.ufrgs.br/asphe/article/view/30047/pdf (Consultado el 16-072020). Lúcio Kreutz y Terciane Ângela Luchese, «Grupos étnicos, pluralidade cultural e políticas públicas na história da educação, no Rio Grande do Sul», Revista Brasileira de História da Educação 11, n. ${ }^{\circ} 1$ (2012): 179-206. http://periodicos.uem.br/ojs/index.php/rbhe/article/view/38511 (Consultado el 16-07-2020).
} 


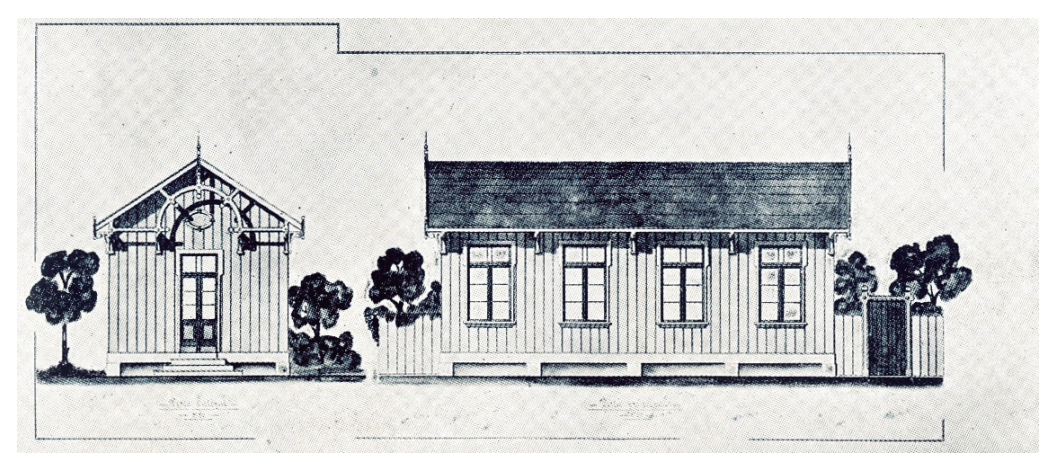

Imagen 5. Planta de Escuela Colonial (1919). ${ }^{36}$

La escuela colonial tendría cinco salas pequeñas, otra más grande y un patio. La construcción también albergaría la casa del maestro, una necesidad que era más frecuente en las zonas rurales, debido a las dificultades en los desplazamientos. Asimismo, se reservaba una sala para el despacho del maestro, al lado de una de las aulas y otros espacios reservados para su morada, como el comedor y el dormitorio. ${ }^{37}$

Partiendo del modelo de 1883, que separaba totalmente el edificio escolar en función del sexo, tanto de los estudiantes como de los maestros, el proyecto estándar de 1899 minimizó esta cuestión, a través de una propuesta simplificada de escuela mixta, que sólo contemplaba la existencia de una sala para clase, de pequeñas dimensiones, sin la previsión de baños u otros espacios -interiores o exteriores- para el desarrollo de la actividad escolar. En 1907, la construcción de un edificio en la capital del Estado, inspirado en el modelo de 1899, seguía la misma propuesta numérica de estudiantes (sesenta), pero con la existencia de un piso inferior, que se podría utilizar para realizar actividades físicas o como casa del maestro. Por su parte, el modelo de escuela primaria colonial aumentó el número de salas, pero mantuvo la residencia del

\footnotetext{
${ }^{36}$ Ildefonso Soares Pinto «Relatório dos Negócios de Obras Públicas do Estado do Rio Grande do Sul», agosto de 1919, s/p. Memorial da Assembleia Legislativa do Estado do Rio Grande do Sul.

37 En 1915 se presentó el proyecto para escuelas aisladas en la ciudad de Bento Gonçalves, diseñado por el ingeniero y exconcejal de la ciudad, João Baptista Pianca. Entre 1925 y 1928, algunas escuelas fueron construidas a partir de este modelo. Véase: Terciane Ângela Luchese y Lúcio Kreutz, «Das escolas de improviso às escolas planejadas: um olhar sobre os espaços escolares da Região Colonial Italiana, Rio Grande do Sul» Revista Brasileira de História da Educação 12, n. ${ }^{\circ} 2$ (2012): 45-76. http:// periodicos.uem.br/ojs/index.php/rbhe/article/view/38787 (Consultado el 16-07-2020).
} 
maestro dentro de las dependencias escolares, principalmente por las dificultades de acceso a estas zonas más asiladas de la capital presentadas por un significativo número de los maestros.

Estas son propuestas representativas de un modelo de escuela que estuvo presente en varios Estados de Brasil, debido a que eran posibles, a pesar de la falta de recursos para implementación de las escuelas graduadas, especialmente, en las ciudades del interior y las zonas rurales. ${ }^{38}$ Posteriormente, estas concepeciones sufrieron importantes cambios a lo largo de la década de 1920, cuando se intensificó en Brasil la discusión en torno a la escuela tradicional y de la Escuela Nueva, que también afectaban a la organización de los espacios y edificios escolares.

\section{ENTRE PERMANENCIAS Y CAMBIOS: UN CONCURSO PARA EDIFICIOS ESCOLARES (1928)}

Entre finales del siglo XIX y las dos primeras decadas del XX, el Estado de Rio Grande do Sul, los problemas relativos a la escuela primaria fueron descritos de forma bastante genérica como pedagógicos y los espacios escolares presentados como una necesidad de adaptación a la pedagogía moderna, la escuela graduada, la coeducación y las exigencias económicas e higiénicas.

\footnotetext{
38 Algunos estudios destacados sobre sobre la implementación de la escuela primaria en Brasil, especialmente los grupos escolares y el análsis principal o secundario sobre sus espacios, son: Rosa Fátima de Souza, Alicerces da Pátria. História da escola primária no Estado de São Paulo (1890-1976), (Campinas: Mercado das Letras, 2009). Luciano Mendes Faria Filho, Dos pardieiros aos palácios: cultura escolar e urbana em Belo Horizonte na Primeira República (Passo Fundo/RS: UPF, 2000). Ester Buffa y Gelson de Almeida Pinto, Arquitetura e Educação: organização do espaço e propostas pedagógicas dos grupos escolares paulistas, 1893-1971 (São Carlos: Brasília: EdUFSCar, INEP, 2002). Marcus Levy Albino Bencostta, «Arquitetura e espaço escolar: o exemplo dos primeiros grupos escolares de Curitiba (1903-1928)» en História da Educação, Arquitetura e Espaço Escolar, ed. Marcus Levy Albino Bencostta (São Paulo: Cortez, 2005), 95-140. Irlen Antonio Gonçalves, «Cultura Escolar, práticas e produção dos grupos escolares em Minas Gerais (1891-1918)» (Tesis doctoral, Universidade Federal de Minas Gerais, 2004). Diana Vidal (ed.), Grupos Escolares: Cultura Escolar Primária e Escolarização da Infância no Brasil (1893-1971), (Campinas: Mercado das Letras, 2006). Crislaine Azevedo, Grupos Escolares em Sergipe - 1911-1930: cultura escolar, civilização e escolarização da infância (Natal: Editora da EDUFRN, 2010). Gladys Mary Ghizoni Teive y Norberto, Dallabrida, A Escola da República: os grupos escolares e a modernização do ensino primário em Santa Catarina (1911-1918), (Campinas, SP: Mercado de Letras, 2011). Sá, Nicamor Palhares y Elizabeth Figueiredo Sá (eds), Revisitando a história da escola primária. Os grupos escolares em Mato Grosso na Primeira República Brasileira. (Cuiabá: Ed. UFMT, 2011). Rosa Fátima de Souza, Vera Lúcia Gaspar da Silva y Elizabeth Figueiredo de Sá, Por uma teoria e uma teoria da escola primaria no Brasil. Investigaçoes comparadas sobre a escola graduada (1870-1930), (Cuiabá: EDUFMT, 2013).
} 
Esta concepción cambia significativamente a lo largo de la década de 1920, que estuvo marcada por el centenario de la indenpendencia de Brasil (1922), los movimientos artísticos modernistas, la elaboración de informes estadísticos sobre la enseñanza pública republicana y la Primeira Conferência Interestadual de Educação Primária, desarrollada entre el 12 de octubre y 16 de noviembre de 1921. Con un total de catorce grupos de estudio, esta última contó con la presencia de representantes de cada Estado del país y de las instituciones educativas privadas. Entre los numerosos temas debatidos, estuvieron la obligatoriedad de enseñanza primaria, la lucha contra el analfabetismo, la articulación de los sistemas estatales de enseñanza y la nacionalización de las escuelas primarias.

La creación de la Associação Brasileira de Educação (ABE), en 1924, generó en Brasil una discusión más intensa entre los plantemientos y los modelos de la escuela tradicional y los de la Escuela Nueva. La fundación de la ABE estuvo a cargo de profesores, maestros, médicos, abogados e ingenieros que tenían como uno de sus principales objetivos sensibilizar a la nación sobre la necesidad de la educación pública, en manos del Estado, en detrimento de la dependencia de otras instancias al margen de este, que aún, después de un siglo de independencia y más de tres décadas de régimen republicano, seguían siendo mayoritarias en la red de escuelas primarias. ${ }^{39}$ Las múltiples y variadas iniciativas desarrolladas por ABE estuvieron en sintonía con las necesidades modernas de la vida espiritual y material del niño y condensaron, adaptándolos a la realidad brasileña, los postulados de la nueva pedagogía. ${ }^{40}$

\footnotetext{
39 Carlos Eduardo Vieira, «Conferências Nacionais de Educação: intelectuais, Estado e discurso educacional (1927-1967)», Educar em Revista 65 (2017): 19-34. https://doi.org/19-34. 10.1590/01044060.53670 (Consultado el 20-03-2020).

40 También cabe destacar el carácter internacionalista de la Escuela Nueva, que estuvo presente desde sus orígenes, a fines del siglo XIX, estimulado por «visitas de educadores, la comunicación y el intercambio de innovaciones y los viajes de pedagogos más conocidos para dar a conocer sus metodos por todo el mundo». Entretanto, es a través de iniciativas institucionales y de asociaciones como del Instituto Jean Jacques Rousseau, en Ginebra/Suiza, creado en 1912 (Oficina Internacional de Educación, dentro del Instituto, creado en 1925) y, la Liga Internacional de Educación Nueva, en 1920, que se canalizaron de forma más concreta la intención del movimiento. María del Mar del Pozo Andrés, «El movimiento pedagógico de la Escuela Nueva», en: Teorías e instituciones contemporáneas de educación, eds. María del Mar del Pozo Andrés et al. (Madrid: Biblioteca Nueva, 2009), 197-216.
} 
En Brasil, lo mismo que en otros muchos países, hubo un número significativo de escuelas que, aunque oficialmente no formaban parte del movimiento de la Escuela Nueva, estaban influidas por ella y habían adoptado parte de sus planteamientos, como fue la perspectiva paidocéntrica de la educación. Aquí y ahora, interesa destacar la busqueda de «nuevos modelos arquitectónicos que permitiesen crear una atmosfera estimulante, para el aprendizaje libre. Se pretendia convertir la escuela en un hogar para el niño».41

Al largo de la decada de 1920, la continuidad de un panorama de insuficiencia de edificios escolares sigue siendo una realidad constante, siendo el alquiler y la adaptación de espacios para las clases primarias una constante en el Estado de Rio Grande do Sul, que estaba organizada en las siguientes instituciones, de acuerdo con el número de estudiantes: colegios elementales (curso primario completo, de cinco o seis años), grupos escolares y clases aisladas. En 1921, de un total de cuarenta y un colegios, que reunían a 14.682 estudiantes, el gobierno del Estado poseía únicamente trece edificios de colegios elementales, otros dieciséis funcionaban en edificios alquilados y cinco en edificios de propiedad de los ayuntamientos; ${ }^{42}$ y de los diez grupos escolares existentes, que albergaban a 819 alumnos, ninguno de sus edificios pertenecía al Estado, pues todos estaban instalados en edificios alquilados -en los municipios de Alfredo Chaves, Canguçu, Santa Vitória do Palmar y São Sebastião do Caí- o en edificios propiedad de los municipios -el de Viamão, São João de Camaquã, São Francisco de Paula, Estrela y Encruzilhada-. ${ }^{43}$

Durante los años siguientes, aparecieron algunas iniciativas aisladas de construcciones escolares. En 1922, se terminaron el Grupo Escolar da Tristeza, ubicado al sur de Porto Alegre, con capacidad para 300

\footnotetext{
${ }^{41}$ Mar del Pozo, «El movimiento pedagógico de la Escuela Nueva», 209.

42 En 1921, la matrícula de la escuela primaria en el Estado de Rio Grande do Sul, según el Informe anual de la Educación Publica, ascendía a 150.009 estudiantes, con frecuencia de 125.371. Cerca del $28 \%$ de los estudiantes estaban matriculados en escuelas subvencionadas por el Estado, el 17\% en escuelas municipales y el 15\% en escuelas particulares. A partir del análisis de los Informes de la Dirección de Instrucción Pública y de la Dirección de Obras Públicas del Estado de Rio Grande do Sul, entre 1907 y 1928, solo nueve edificios escolares de educación primaria fueron emprendidos por el poder público estatal. Ermel, «Arquitetura escolar e patrimônio histórico-educativo», 117.

43 Protásio Alves «Relatório da Secretaria do Interior e do Exterior do Estado do Rio Grande do Sul, Diretoria de Instrução Pública», agosto de 1921, p. 189. Memorial da Assembleia Legislativa do Estado do Rio Grande do Sul.
} 
alumnos, distribuidos en seis aulas, y una pequeña clase aislada en un edificio de madera en el municipio Gravataí, cerca de las minas de carbón del Estado. En 1928, se iniciaron las obras de otros tres edificios escolares de nueva planta: la Escuela Aislada de Chácara das Bananeiras, en la ciudad de Porto Alegre, y dos edificios para escuelas primarias en las ciudades de Passo Fundo y Cruz Alta, que siguieron el mismo proyecto. Ese mismo año, el Decreto numero 3.981, de 5 de enero de 1928, estableció un presupuesto de cuatrocientos mil reales para los edificios escolares; doscientos cincuenta mil destinados a la construcción y ciento cincuenta mil reservados para asegurar la conservación de los existentes.

También en 1928, en respuesta a la escasez de edificios adecuados para la escuela primaria, el gobierno del Estado lanzó un concurso de proyectos de construcción de edificios escolares urbanos y rurales, que fue publicado en la prensa oficial a través del periódico A Federação y reproducido, al año siguiente, en el Informe anual de la Dirección de Educación Publica. ${ }^{44}$ De las catorce cláusulas del concurso, cinco fueron cuestiones burocráticas -forma de presentación (plan general, planos parciales, fachadas y secciones), memoria descriptiva y justificativa, firma reconocida, comisión técnica evaluadora y premiación- y nueve demandas específicas relativas a los espacios.

Estas últimas contemplaron distintos aspectos, que se pueden sintetizar del siguiente modo. En primer lugar, cabe señalar la permanencia de tres modelos de escuela primaria en el Estado: colegios elementales, grupos escolares y aulas aisladas -el modelo el más simple, con apenas la prescripción de una o dos salas, con capacidad para cincuenta estudiantes cada una, y con la posibilidad de un espacio reservado para la residencia del maestro-. En segundo lugar, se establecieron algunas normas de higiene, como la existencia de un vestidor y toilette con baño, un

\footnotetext{
${ }^{44}$ En este mismo año, el gobierno del Estado invirtió en la supervisión de los edificios escolares existentes. Con este fin, preparó un cuestionario sobre las condiciones de los terrenos y edificios de la escuela, que debería ser respondido por un equipo de inspectores, y se observaron cuidadosamente las condiciones del terreno, la construcción y los complementos. En este último, podrían o no estar directamente relacionados con la escuela, como la existencia o no de «patios de juego o patios de recreo para niños o niñas (cubiertos con arena, etc.); patio de recreo; jardínes y arboles; pabellón del gimnasio; granja escolar; plaza deportiva (en pueblo o aldea); talleres, etc». Oswaldo Aranha «Relatório da Secretaria do Interior e do Exterior do Estado do Rio Grande do Sul, Diretoria de Instrução Pública», agosto de 1929, pp. 25-27. Memorial da Assembleia Legislativa do Rio Grande do Sul.
} 
baño para cada grupo de quince niñas o treinta niños y un urinario para cada grupo de quince estudiantes. En tercer término, se apostó por planteamientos pedagógicos generalistas y consensuados, pero dando la posibilidad a los concursantes de presentar otras propuestas pedagógicas.

Las propuestas para colegios elementales y grupos escolares tuvieron que presentar dos versiones, una para doscientos estudiantes y otra para mil, ambas con la posibilidad de ampliación, sin comprometer la estética o los principios pedagógicos. En cuanto al entorno, los edificios deberían estar aislados del vecindario y no tener más de dos plantas, con ventilación eficiente e iluminación directa. A estos dos modelos de escuela primaria se incorporaron elementos novedosos, tales como: aulas rectangulares, que deberían tener esquinas redondeadas, siendo que la planta debería diseñarse de modo que se pudiera aumentar el número de habitaciones, sin perjuicio de la estética y la higiene del edificio. Otro aspecto es la variante de paredes móviles, que servirían para la transformación de cuatro o más salas en un salón de actos o auditorio, así como al contrario, la subdivisión de dos o tres salas para que cada una pueda albergar solo a veinticinco estudiantes. También estaba previsto, en ambos casos, la existencia de un pabellón cubierto, a modo de gimnasio.

La incorporación de una variedad de salas también fue un elemento destacado en el concurso: de espera, administración, biblioteca, museo didáctico, trabajos manuales, consultorio de un médico, atención odontológica, auditorio -o salón de ceremonias-. Finalmente, cabe destacar la exigencia de establecer espacios para la futura instalación del cine y la radio escolares, que era intención que se generalizaran en la red escolar pública del país. Sobre estos dos últimos puntos, se pueden destacar varias iniciativas. La primera es el proyecto pionero de Cinema Escolar en Brasil, desarrollado entre 1916 y 1918, bajo la dirección de dos inspectores escolares de la red pública municipal de Río de Janeiro: Fábio Lopes dos Santos, un médico higienista, y José Venerando da Graça Sobrinho, un funcionario municipal. El objetivo giró en torno a la producción de lo que llamaron cintas pedagógicas y la idea del cine educativo, que se entendió como el desarrollo de películas con los propios estudiantes. ${ }^{45}$

\footnotetext{
45 Amália da M. Mendonça Ferreira, «O cinema escolar na história da educação brasileira. A sua ressignificação através da análise do discurso» (Trabajo de fin de Máster, Universidade Federal Fluminense, 2004).
} 
La segunda propuesta destacable es la realizada por Edgar Roquette-Pinto, Jonathas Serrano y Venâncio Filho, que, partiendo del discurso escolanovista, entendieron que el cine podía ser una poderosa herramienta educativa auxiliar al servicio del maestro para desarrollar su tarea, lo mismo que lo eran la biblioteca, el periódico, el banco escolar y el club agrícola.

La tercera corrió a cargo de la Associação Brasileira de Educação (ABE), cuyos estatutos recogían entre sus objetivos el de «facilitar o desenvolvimento do cinema educativo, de bibliotecas infantis e de outras instituições auxiliares ao ensino». ${ }^{46}$ Durante el transcurso de la primera Conferência Nacional de Educação (CNE), promovida por la $\mathrm{ABE}$, que tuvo lugar en la ciudad de Curitiba/Paraná, en 1927, una de las ponencias presentadas destacó por tratar las directirces generales de la Escuela Nueva Brasileira: una escuela del trabajo, social, saludable, cientifica y práctica, ajustada a las necesidades y posibilidades del momento, capaz de desarrollar las aptitudes de los alumnos y orientada hacia el mundo laboral. De modo más específico, también fueron abordados temas sobre el sentido de las relaciones del niño con su medio, enfatizando las posiblidades educativas que las realidades, los recursos y los entornos sociales de cada Estado ofrecían a las escuelas. ${ }^{47} \mathrm{El}$ cine, la literatura infantil, las pinacotecas, el teatro, el museo y la prensa escolar fueron temas abordados por los conferenciantes. Así, por ejemplo, el cine fue analizado por Maria Luiza Camargo de Azevedo, que lo describió como una espada de doble filo, esto es que, al mismo tiempo que educa, también podría corromper a los niños, por lo que era necesario que los maestros establecieran criterios claros para su uso pedagógico; por su parte, Américo Xavier Monteiro de Barros, otro de los ponentes, defendió las contribuciones y la enseñanza ilustrada que había posibilitado el cinematógrafo escolar en los grandes centros, aconsejando su extensión, de acuerdo con las posibilidades de cada Estado. ${ }^{48}$

\footnotetext{
46 Vieira, «Conferências Nacionais de Educação», 25.

47 Susana da Costa Ferreira, "A I Conferência Nacional de Educação (Contribuição para o estudo das origens da Escola Nova no Brasil)», Instituto Nacional de Estudos e Pesquisas Educacionais (INEP) 6 (1993): 37-38.

${ }^{48}$ Ferreira, "A I Conferência Nacional de Educação».
} 
En relación con la radio, la primera estación registrada en Brasil fue la establecida por Roquete-Pinto, en el año 1922, llamada Radio Sociedade de Rio de Janeiro. ${ }^{49}$ A partir de 1924, se acercó a una perspectiva más popular de la educación, transmitiendo clases en francés, portugués, geografía, historia brasileña, higiene, silvicultura, química, historia natural y física, así como emisiones de conciertos y representaciones teatrales. Según su propuesta, cada estado tendría su escuela de radio, siendo posibles también las escuelas de radio municipales..$^{50}$

\section{CONSIDERACIONES FINALES}

Las prescripciones generales sobre espacios escolares vigentes durante finales del siglo XIX y las primeras tres décadas del XX, tanto en el contexto internacional como en la realidad particular de Rio Grande do Sul, revelan aspectos sobre permanencias y transformaciones signficativas de las ideas y las prácticas vinculadas a los edificios y recintos de las instituciones educativas de carácter público. Así, se percibe un tránsito de la inexistencia y/o inadecuación de los edificios de las escuelas urbanas y rurales, las dificuldades de implementación de la escuela graduada y las nociones higinistas, hacia una adaptación gradual del gobierno del Estado a los nuevos paradigmas de la Pedagogía, especialmente de la escuela activa o Escuela Nueva.

De los ejemplos que hemos elegido para ilustrar este estudio, el primer modelo de edificio escolar (1883) reproducía tres elementos básicos de la escuela primaria en su configuración inicial, o sea, el aula como espacio educativo central, una total separación por sexos y la residencia del maestro en el edificio escolar. Pocos años después, el modelo estándar de escuela de 1899 y su edificación en forma de una clase aislada en la Capital, en 1907, torna visible un proyecto destinado a un número reducido de estudiantes, una clase unitaria mixta, sin previsión de

\footnotetext{
49 José Silvério Baia Horta, "Histórico do rádio educativo no Brasil (1922-1970)», Cadernos da PUC-Rio (Tópicos em Educação/serie Letras e Artes, Rio de Janeiro, 1972).

${ }^{50}$ En la Reforma de la Educación Pública, implementada por Fernando de Azevedo, en Río de Janeiro (1927-1930), el cine se introdujo como un instrumento educativo, por lo que fue obligatorio tener salas para proyección fija y animada, cuando la escuela operara en su propio edificio. Sin embargo, no fue hasta 1937 cuando, a propuesta del ministro Capanema, el Instituto Nacional de Cine Educativo (INCE) empezó a considerarse como un servicio público. En 1937, junto con el cine educativo, se creó el programa de radiodifusión educativa.
} 
sanitarios y con posibilidad de morada del maestro o la existencia de un área cubierta para actividades físicas en días de lluvia. Destaca una preocupación con la posición solar y la entrada de ventilación, así como la protección de los estudiantes del entorno de la ciudad a través de la valla de la pared.

Una constante en las discusiones sobre las escuelas primarias fue su instalación en las zonas rurales y algunas cuestiones aparejadas, como los problemas de transporte, la morada del maestro, las dimensiones que habría de tener y los materiales que utilizar para la construcción. Eran frecuentes las pequeñas instalaciones de madera, financiadas por el ayuntamiento o la donación por la comunidad en las regiones desproveídas por el Gobierno del Estado. El modelo presentado en 1919, también en madera, contemplaba únicamente el espacio del aula, conforme al modelo de 1899, pero con una descripción detallada de los espacios destinados a la residencia del maestro.

Asimismo, se perciben permancias y cambios en la proyección de edificios prescritos en el concurso de 1928, que inaugura una proyección compleja en torno a las ideas sobre los espacios escolares en Rio Grande do Sul. La relación entre la residencia del maestro y el edificio escolar, previsto en los modelos anteriores, permanece solamente en la modalidad de clases aisladas. La separación del edificio por sexo, conforme el modelo de 1883, está totalmente ausente de las cláusulas del concurso de 1928. Se mantuvieron, no obstante, los tres principales tipos de escuelas primarias existentes en el Estado: clase aislada, grupo escolar y colegios elementales, siendo que la enseñanza graduada debería ser implementada en estos dos últimos casos, con variedad de tamaño, de acuerdo con la demanda escolar, pero con una capacidad máxima para mil estudiantes.

La relación de la escuela con su entorno, así como la representación política, social y cultural de las construcciones que se incorporaron a lo largo de la Primera República brasileña se concretó en la prescripción del aislamiento de los edificios escolares del resto del vecindario y en el establecimiento de dos plantas para albergar grupos escolares y colegios elementales. La preocupación higienista continuó centrada en los espacios ventilados y la entrada adecuada de luz, pero con aumento de elementos sanitarios, como el vestidor, el toilette con baño, los urinarios 
y aseos en número proporcional al de estudiantes. Los espacios específicos para las actividades físicas, no descritos en modelos anteriores, ahora estuvieron previstos en forma de pabellón o gimnasio cubierto; no ocurrió así con los espacios para las actividades al aire libre, centrales en las prerrogativas de la Escuela Nueva, pero ausentes en las descripciones de los proyectos.

Desde otro punto de vista, consideramos importantes los elementos que, en esa época, fueron incorporados como novedosos en el escenario brasileño y, especialmente, en Rio Grande do Sul, como las esquinas redondeadas y los espacios adaptables para el aumento y/o disminuición de los tamaños de las salas y del gimnasio. La obligación de que existieran salas variadas, laboratorios y la previsión de otras dependencias para la futura instalación del cine y la radio escolares eliminaron parte de la centralidad del aula, en beneficio de otros espacios educativos adaptados a las necesidades y posibilidades físicas de los niños y más propicios para generar un ambiente capaz de estimularlos y alimentar su curiosidad.

En Rio Grande do Sul, el concurso de edificios escolares, al parecer, no tuvo repercusión posterior a su publicación en la prensa, en 1928, y su reproducción en el Informe de Educación Pública, de 1929. En Brasil, la década de 1930 se inició con importantes cambios políticos -finalizó la Primera República- y educativos -se creó el Ministério de Educação e Saúde Pública, en 1931-. Poco tiempo después, en 1932, la ABE y sus miembros lanzaron el Manifesto dos pioneros da Escola Nova, que inauguró una década de difusión de las ideas de la Escuela Nueva en Brasil, a través de publicaciones, como las de Lourenço Filho ${ }^{51}$, y prácticas llevadas a cabo por importantes renovadores, como Fernando de Azevedo y Anisio Teixeira, a través de las reformas que emprendieron.

El plan de construcciones de edificios escolares emprendido por el Gobierno del Estado de Rio Grande do Sul siguió un ritmo lento y con características semejantes a los modelos más tradicionales de las decadas anteriores, pero con ligero impulso en número, ya durante el periodo del Estado Novo (1937-1945). Consideramos que, en el período

\footnotetext{
${ }^{51}$ Las ideas de Lourenço Filho serán ampliamente difundidas en plan nacional a través de su obra «Introdução ao estudo da Escola Nova», editora Melhoramentos, 1930.
} 
analizado, en el Estado de Rio Grande do Sul de Brasil, lo mismo que en otros países occidentales, persistió un modelo de escuela en el que el aula era el elemento central, pero permeado, progresivamente, en distinta medida, y casi siempre de manera puntual, por elementos e ideas de la Escuela Nueva y otras corrientes pedagógicas que pretendían modernizar la enseñanza.

\section{Nota sobre autora}

Tatiane de Freitas Ermel es investigadora del Programa de Atracción de Talento de la Comunidad de Madrid (modalidad jóvenes doctores) en el Departamento de Estudios Educativos de la Universidad Complutense de Madrid. Fue profesora de la Universidade Regional Integrada do Alto Uruguai e Missões/Brasil y becaria en la categoria Joven Investigador del Conselho Nacional de Desenvolvimento Científico e Tecnológico CNPq/Brasil (2017-2018). Doctora en Educación - Pontifícia Universidade Católica do Rio Grande do Sul/Brasil (2017), seleccionada al Prêmio Nacional - Tesis Doctoral Capes 2018/Brasil. Las líneas de investigación especialmente desarrolladas son: espacios educativos y arquitectura escolar; prensa pedagógica; circulación de las ideas pedagógicas. Es miembro del grupo de investigación: Cultura Cívica y Políticas Educativas y Connecting History of Education Working Group.

\section{REFERENCIAS}

Arruda, Ângelo Marques Vieira de, «Arquitetura dos edifícios da escola pública no Brasil (1870-1930): construindo os espaços para a educação», PhD diss., Universidade Federal do Mato Grosso do Sul, 2010.

Azevedo, Crislaine. Grupos Escolares em Sergipe - 1911-1930: cultura escolar, civilização e escolarização da infância. Natal: Editora da EDUFRN, 2010.

Baltar, Francisca Maria Teresa dos Reis. "Arquitetura de escolas no século 19: primeiras escolas construídas no Brasil», Revista História da Educação 10 (2001): 53-84. https://seer.ufrgs.br/asphe/article/view/30525/pdf

Bastos, Maria Helena Camara "A educação como espetáculo». En Histórias e Memórias da Educação no Brasil, editado por Maria Stephanou y Maria Helena Camara, 116-131. Petrópolis: Vozes, 2005.

Bencostta, Marcus Levy Albino. «Arquitetura e espaço escolar: o exemplo dos primeiros grupos escolares de Curitiba (1903-1928)» en História da Educação, 
Arquitetura e Espaço Escolar, editado por Marcus Levy Albino Bencostta, 95140. São Paulo: Cortez, 2005.

Bencostta, Marcus Levy y Marina Fernandes Braga. «História e Arquitetura escolar: a experiência dos regulamentos franceses e brasileiros para os edifícios escolares (1890-1910)». Revista Linhas 12, n. ${ }^{\circ} 1$ (2011): 51-72.

Bernard, Henry. School Architecture: contributions to the improvement of school-houses. Cincinati: Published by H.W. Derby and Co., 1855.

Buffa, Ester y Gelson de Almeida Pinto. Arquitetura e Educação: organização do espaço e propostas pedagógicas dos grupos escolares paulistas, 1893-1971. São Carlos: Brasília: EdUFSCar, INEP, 2002.

Chervel, André. La culture scolaire: une approche historique. Paris: Belin, 1998.

Cunha, Marcus Vinicius da. "O legado da Escola Nova e a dimensão utópica da educação», en O curso de Lourenço Filho na Escola Normal do Ceará (19221923): as normalistas e a pedagogia da escola nova, editado por Maria Helena Camara Bastos y Maria Juraci Maia Cavalcante, 339-358. Campinas: Alínea, 2009.

Cury, Carlos Roberto Jamil. «A educação e a primeira constituinte republicana» en Educação nas constituições brasileiras 1823-1988, editado por Osmar Fávero, 69-80. Campinas: Autores Associados, 1996.

Ermel, Tatiane de Freitas. "Arquitetura escolar e patrimônio histórico-educativo: os edificios para a escola primária pública no Rio Grande do Sul (19071928) ", PhD diss., Pontifícia Universidade Católica do Rio Grande do Sul, 2017.

Ermel, Tatiane de Freitas. «Transfigurações no tempo e no espaço: Aula Isolada Campo da Redenção em Porto Alegre/RS (1907-2016)», Revista Brasileira de História da Educação 18, n. 48 (2018): 1-32.

Escolano Benito, Agustín. Tiempos y espacios para la escuela. Ensayos Históricos. Madrid: Biblioteca Nueva, 2000.

Faria Filho, Luciano Mendes et al. "A Cultura Escolar como categoria de análise e como campo de investigação na história da educação brasileira». Revista Educação e Pesquisa 30, n. ${ }^{\circ} 1$ (2004): 139-159.

Faria Filho, Luciano Mendes. Dos pardieiros aos palácios: cultura escolar e urbana em Belo Horizonte na Primeira República. Passo Fundo/RS: UPF, 2000.

Ferreira, Amália da M. Mendonça. «O cinema escolar na história da educação brasileira. A sua ressignificação através da análise do discurso». PhD diss., Universidade Federal Fluminense, 2004.

Ferreira, Susana da Costa. "A I Conferência Nacional de Educação (Contribuição para o estudo das origens da Escola Nova no Brasil)», Instituto Nacional de Estudos e Pesquisas Educacionais (INEP), 6 (1993): 37-38. 
Godínez Visedo, José Miguel. «Legislación comentada sobre espacios y construcciones escolares de los centros públicos primarios en España. De 1825 a 1991» Anales de Pedagogía 11 (1993): 229-243.

Gonçalves, Irlen Antonio. «Cultura Escolar, práticas e produção dos grupos escolares em Minas Gerais (1891-1918)». PhD diss., Universidade Federal de Minas Gerais, 2004.

Horta, José Silvério Baia. Histórico do rádio educativo no Brasil (1922-1970), Cadernos da PUC-Rio, Rio de Janeiro: Tópicos em Educação/serie Letras e Artes, 1972.

Ibarra, Carlos Ortega. «Ciencia y revolución en la arquitectura escolar. Ciudad de México (1910-1920)». Revista Ciencia y Desarrollo (2012): 39-42.

Julia, Dominique. «La culture scolaire comme objet historique». Paedagogica Historica, 31 (1995): 353-382. https://doi.org/10.1080/00309230.1995.11434 853

Kreutz, Lúcio. «A representação de identidade nacional em escolas da imigração alemã no Rio Grande do Sul», Revista História da Educação 3, n. ${ }^{\circ} 5$ (1999): 141-164. https://seer.ufrgs.br/asphe/article/view/30047/pdf

Kreutz, Lúcio y Terciane Ângela Luchese. «Grupos étnicos, pluralidade cultural e políticas públicas na história da educação, no Rio Grande do Sul», Revista Brasileira de História da Educação 11, n. ${ }^{\circ}$ (2012): 179-206. http://periodicos.uem.br/ojs/index.php/rbhe/article/view/38511

Luchese, Terciane Ângela y Lúcio Kreutz. «Das escolas de improviso às escolas planejadas: um olhar sobre os espaços escolares da Região Colonial Italiana, Rio Grande do Sul» Revista Brasileira de História da Educação 12, n. ${ }^{\circ} 2$ (2012): 45-76. http://periodicos.uem.br/ojs/index.php/rbhe/article/ view/38787

Manique da Silva, Carlos. Escolas belas ou espaços sãos? Uma análise histórica sobre arquitetura escolar portuguesa (1860-1920). Lisboa: Instituto de Inovação Educacional, 2002.

Pozo Andrés, María del Mar del «El movimiento pedagógico de la Escuela Nueva», en Teorías e instituciones contemporáneas de educación, editado por María del Mar del Pozo Andrés et al., 197-216. Madrid: Biblioteca Nueva, 2009.

Recalde, Héctor. El Primer Congreso Pedagógico/1 (1882). Biblioteca Política Argentina: Centro Editor de América Latina, 1987.

Sá, Nicamor Palhares y Elizabeth Figueiredo Sá (eds). Revisitando a história da escola primária. Os grupos escolares em Mato Grosso na Primeira República Brasileira. Cuiabá: Ed. UFMT, 2011.

Santiago, Zilsa Maria Pinto. Arquitetura e Instrução Pública: a reforma de 1922, concepção de espaços arquitetônicos e formação dos primeiros grupos escolares do Ceará. Ceará: Edições Universidade Federal do Ceará, 2017. 
Schueler, Alessandra Frota y Irma Rizzini. «Entre becos, morros e trilhos: expansão da escola primária na cidade do Rio de Janeiro (1870-1906)» Cadernos de História da Educação 18, n. ${ }^{\circ} 1$ (2019): 160-175. https://doi.org/10.14393/ che-v18n1-2019-9

Souza, Rosa Fátima de y Vera Teresa Valdemarin (ed.). A Cultura Escolar em Debate: questões conceituais, metodológicas e desafios para a pesquisa. Campinas: Autores Associados, 2005.

Souza, Rosa Fátima de, Vera Lúcia Gaspar da Silva y Elizabeth Figueiredo de Sá (eds,). Por uma teoria e uma teoria da escola primaria no Brasil. Investigaçoes comparadas sobre a escola graduada (1870-1930). Cuiabá: EDUFMT, 2013.

Souza, Rosa Fátima de. Alicerces da Pátria. História da escola primária no Estado de São Paulo (1890-1976), Campinas: Mercado das Letras, 2009.

Souza, Rosa Fátima de. Tempos de Civilização: a implantação da Escola Primaria Graduada no Estado de São Paulo (1890-1910). São Paulo: UNESP, 1998.

Teive, Gladys Mary Ghizoni y Norberto, Dallabrida. A Escola da República: os grupos escolares e a modernização do ensino primário em Santa Catarina (1911-1918). Campinas: Mercado de Letras, 2011.

Tubeuf, Georges. Traité d'architecture théorique et pratique. Tome IV, Tipes de constructions diverses: edifices publics et divers. Paris: Georges Fancho Editeur, 1890.

Upitis, Rena. "School Architecture and Complexity», Complicity: An International Journal of Complexity and Education, 1 (2004): 19-38. https://doi. org/10.29173/cmplct8713

Vidal, Diana (ed.). Grupos Escolares: Cultura Escolar Primária e Escolarização da Infância no Brasil (1893-1971). Campinas: Mercado das Letras, 2006.

Vidal, Diana. «Sobre cultura escolar e História da Educação: questões para debate». En História das culturas escolares no Brasil, editado por Diana Vidal y Cleonara Maria Schwatz, 13-35, Vitória: EDUFES, 2010.

Vieira, Carlos Eduardo. "Conferências Nacionais de Educação: intelectuais, Estado e discurso educacional (1927-1967)», Educar em Revista, 65 (2017): 19-34. https://doi.org/19-34. 10.1590/0104-4060.53670

Vincent, Guy (ed.). L'éducation prisonnière de la forme scolaire? Scolarisation et socialisation dans les sociétés industrielles. Lyon: Presses Universitaires de Lyon, 1994.

Viñao Frago, Antonio. "Do espaço escolar e da escola como lugar: propostas e questões». En Currículo, espaço subjetividade: a arquitetura como programa, editado por Antonio Viñao Frago y Agustín Benito Escolano, 59-140. Rio de Janeiro: DP\&A, 1998.

Viñao Frago, Antonio. Sistemas Educativos, culturas escolares y reformas. Razones y propuestas educativas. Madrid: Ediciones Morata, 2002. 
Weisser, Amy S. «Little Red School House, What Now? Two Centuries of American Public School Architecture», Journal of Planning History 3 (2006): 196-217. https://doi.org/10.1177/1538513206289223

Werle, Flávia Obino Corrêa. «Instrução Pública e Configuração do Mundo Urbano». História da Educação, 18 (2005): 83-95. https://seer.ufrgs.br/asphe/ article/view/29128/pdf

Wollf, Silvia Ferreira Santos. Escolas para a República: os primeiros passos da Arquitetura das escolas públicas paulistas. São Paulo: EDUSP, 2010. 